\title{
Une vision de l'excellence en pratique pharmaceutique
}

par Patricia Macgregor

$E^{n}$ n 2008, la Société canadienne des pharmaciens d'hôpitaux (SCPH) a lancé son projet SCPH 2015, qui propose une vision de l'excellence en pratique pharmaceutique, mettant ses membres de partout au pays au défi de mettre en valeur la pratique pharmaceutique dans le but d'améliorer les soins aux patients et les résultats pharmacothérapeutiques. Aujourd'hui, alors qu'il ne reste que 18 mois à cette initiative axée sur le patient, nous pouvons parler fièrement des nombreuses actions entreprises jusqu'à maintenant qui portent sur les 36 objectifs, fondés sur des données probantes, compris dans les six buts clés du projet SCPH 2015.

On a demandé aux leaders de pharmacie hospitalière de s'engager à soutenir le rôle de leurs pharmaciens pour la mise en œuvre du projet SCPH 2015 et à faire part des réalisations à leurs collègues partout au pays. L'expertise dont nous ont fait profiter des membres appartenant à toutes les sections de la SCPH a produit une accumulation incroyable de ressources, notamment 30 récits de succès, 34 affiches virtuelles, 3 trousses à outils et 10 webinaires archivés. Toutes les ressources sont publiées sur le site Web de la SCPH (www.cshp.ca/cshp2015). Les pharmaciens ont indiqué que les trousses sont des ressources précieuses au moment de la mise en œuvre de nouveaux processus. De plus, le taux élevé de participation aux webinaires de la SCPH témoigne de la valeur que la Société apporte grâce à ce type de partage d'expertise. Les communications reçues de collègues ailleurs dans le monde révèlent aussi la portée de la diffusion des connaissances. En plus des ressources du site Web, on compte la Conférence sur la pratique professionnelle (CPP) et le Séminaire de gestion en pharmacie Harrison parmi les occasions récentes de diffusion des connaissances émanant du projet SCPH 2015.

En juin 2013, les directeurs de pharmacie hospitalière ont été sondés afin de déterminer quels sont leurs besoins en lien au projet SCPH 2015. Les résultats du sondage, qui ont été transmis pendant la CPP de 2014, ont permis de définir 10 objectifs clés du projet SCPH 2015 pour lesquels les directeurs souhaitent un appui. Les trois objectifs prioritaires sont : des programmes à l'échelle de l'organisation visant à étudier l'utilisation sécuritaire des médicaments, la prise en charge et la surveillance par les pharmaciens de la pharmacothérapie des patients hospitalisés, et l'application de la technologie des codes-barres. Les résultats faisaient également ressortir d'autres priorités qui nécessitent elles aussi l'attention des directeurs de pharmacie.

L'évaluation des indicateurs clés de rendement représente un aspect important permettant de juger de la valeur. Les outils élaborés pour le projet SCPH 2015 offrent d'excellentes occasions aux pharmaciens de faire part de la progression dans leur cheminement vers l'excellence, de souligner et de célébrer les réussites, et de faire connaitre aux autres la proposition de valeur des pharmaciens pour ce qui touche aux résultats pharmacothérapeutiques. Pendant la CPP, on a de plus mis de l'avant les tableaux de progression du projet SCPH 2015 dont les données ont été colligées par l'intermédiaire du sondage sur les pharmacies hospitalières canadiennes. Ces tableaux de bord pratiques permettent de comparer les progrès d'un service de pharmacie hospitalière aux réalisations régionales des objectifs du projet SCPH 2015. Puisque le sondage sur les pharmacies hospitalières canadiennes est réalisé tous les deux ans, le prochain (au printemps 2014) représente donc la dernière chance d'évaluer la progression vers l'atteinte des objectifs du projet SCPH 2015 par cette voie.

En vue de l'aboutissement du projet SCPH 2015, la SCPH réalise un montage vidéo, amorcé pendant la CPP de cette année, afin de mettre en lumière les récits de pharmaciens illustrant l'influence qu'a eue le projet SCPH 2015 sur la pratique de la pharmacie et sur les soins aux patients.

Alors que le projet SCPH 2015 tire à sa fin, j'invite la communauté des pharmaciens et chacun de ses membres à s'approprier davantage cette vision de l'excellence en pratique, à assister à la CPP en février 2015 pour ovationner à la tombée du rideau sur le projet SCPH 2015 et à améliorer les soins aux patients et les résultats pharmacothérapeutiques grâce à leurs efforts soutenus pour faire avancer la pratique de la pharmacie.

Patricia Macgregor, B. Sc., R. Ph., M. R. Pharm. S., M. H. Sc., C.H.E., est présidente et agente de liaison pour la vision de la Société canadienne des pharmaciens d'hôpitaux. 